FSU-HEP-951229

UIOWA-95-07

\title{
MULTIPLE PARTON EMISSION EFFECTS IN NEXT-TO-LEADING-ORDER DIRECT PHOTON PRODUCTION
}

\author{
Howard Baer ${ }^{1}$ and Mary Hall Reno ${ }^{2}$ \\ ${ }^{1}$ Department of Physics, Florida State University, Tallahassee, FL 32306 USA \\ ${ }^{2}$ Department of Physics and Astronomy, University of Iowa, Iowa City, IA 52242 USA
}

(October 16, 2018)

\begin{abstract}
A recent global analysis of direct photon production at hadron collider and fixed target experiments has noted a disturbing trend of disagreement between next-to-leading-order (NLO) calculations and data. The conjecture has been made that the discrepancy is due to explicit multiple parton emission effects which are not accounted for in the theoretical calculations. We investigate this problem by merging a NLO calculation of direct photon production with extra multiple parton emissions via the parton shower (PS) algorithm. Our calculation maintains the integrity of the underlying NLO calculation while avoiding ambiguities due to double counting of multiple parton emissions. We find that the NLO+PS calculation can account for much of the theory/CDF data discrepancy at $\sqrt{s}=1.8 \mathrm{TeV}$. It can also account for much of the theory/UA2 discrepancy if a very large virtuality is assumed to initiate the initial state parton shower. For lower energy data sets $($ e.g. $\sqrt{s}<63$ $\mathrm{GeV}), \mathrm{NLO}+\mathrm{PS}$ calculations alone cannot account for the data/theory discrepancy, so that some additional non-perturbative $k_{T}$ smearing is needed.
\end{abstract}

PACS numbers: 12.38.Bx, 12.38.Qk, 13.85.Qk, 14.80.Am 


\section{INTRODUCTION}

Direct photon production [1] 5] in hadronic collisions has long been recognized as an important testing ground for perturbative QCD since many of the ambiguities involved in measuring jets are not present when analyzing photons. Direct photon production in lowest order QCD takes place via annihilation and Compton scattering Feynman graphs. Since the Compton graph involves initial state gluon-quark scattering, measurements of direct photon events can serve as important constraints in the determination of the gluon parton distribution function [5]. For such a program to proceed, the greater precision involved in next-to-leading order (NLO) QCD calculations for the hard scattering are used. NLO calculations for parton+parton $\rightarrow \gamma X$ have been performed both analytically [6] and in a Monte Carlo framework [7].

A recent global analysis of direct photon production in hadron collisions has noted a discrepancy between NLO calculations and a large array of data for the transverse momentum $p_{T}$ distributions of the photon [8]. Characteristically, in both fixed target and collider experiments, there is an experimental excess of photons at low transverse momentum. Several possible explanations have been put forth to resolve the discrepancy. These include $i$ ) improved (NLO) treatment of bremsstrahlung contributions [9] and isolation criteria [10], ii) modifying gluon distribution functions and QCD scale choices to improve the data/theory agreement [10], or usage of alternative parton distribution functions (PDF's) [11], and iii) invoking additional partonic $k_{T}$ smearing effects [8]. The latter case comes in two different guises: extra partonic $k_{T}$ can come from non-perturbative effects from parton binding and intrinsic transverse momentum, or from additional hard multiple parton emissions which can be calculated or modeled in perturbative QCD. The non-perturbative effects are generally implemented as Gaussian smearing in an attempt to match the data. The perturbative multiple gluon emission effects can be implemented via even higher (but fixed) order perturbative calculations, via multiple gluon resummation techniques, or via the parton shower (PS) algorithm [12,13]. The resummation and PS approaches both involve approximate all orders perturbative QCD effects.

In this paper, we explore the extent to which the direct photon data can be explained by combining a NLO QCD calculation with multiple parton emission via the parton shower algorithm. In doing so, we follow generally the prescription outlined in Ref. [14], where NLO $W$ and $Z$ boson production were merged with parton showers. In these calculations, Owens' method of phase space slicing is used to evaluate the NLO cross sections [15]. This method lends itself to a direct implementation of parton showers wherein a potential problem of double counting multiple parton emissions can be avoided. We show that our implementation of showering with the NLO QCD calculation yields an excess of events at low $p_{T}$ relative to the unshowered NLO result at the Fermilab Tevatron and CERN Sp $\bar{p} \mathrm{~S}$ energies, qualitatively accounting for the discrepancy between theory and experiment. Additional

non-perturbative smearing is required for lower energies characteristic of the CERN ISR or fixed target experiments. 


\section{CALCULATIONAL PROCEDURE}

Central to our calculation of direct photon production is the numerical integration of phase space via Monte Carlo methods [7]. One begins by evaluating the $\mathcal{O}\left(\alpha \alpha_{s}\right)$ and $\mathcal{O}\left(\alpha \alpha_{s}^{2}\right)$ direct photon production subprocess Feynman graphs, including bremsstrahlung corrections to $q \bar{q} \rightarrow q \bar{q}$, etc. Dimensional regularization is used here for ultraviolet, soft and collinear singularities. The four-momenta for the $2 \rightarrow 2$ subprocess are labeled according to, for instance, $g\left(p_{1}\right)+q\left(p_{2}\right) \rightarrow \gamma\left(p_{3}\right)+q\left(p_{4}\right)$; similarly, for $2 \rightarrow 3$ subprocesses, we use $g\left(p_{1}\right)+$ $q\left(p_{2}\right) \rightarrow \gamma\left(p_{3}\right)+q\left(p_{4}\right)+g\left(p_{5}\right)$, etc. Ultraviolet singularities are renormalized using the $\overline{\mathrm{MS}}$ prescription [6]. Collinear singularities are factorized and then absorbed into parton distribution functions (PDF's) or fragmentation functions. Soft singularities are canceled between $2 \rightarrow 3$ graphs and $2 \rightarrow 2$ graphs. At this point, all cross section contributions are finite, so that numerical predictions can be made.

What is peculiar to the Monte Carlo method of NLO calculation used here is that the phase space integrations are done partly analytically, and partly numerically. The boundary between numerical and analytical methods is chosen by selecting two theoretical cutoffs to demarcate the collinear and soft regimes. If any invariant quantity $t_{i j} \equiv\left(p_{i}-p_{j}\right)^{2}$ from the $2 \rightarrow 3$ subprocess has a value $\left|t_{i j}\right|<\delta_{c} s_{12}$, where $s_{i j}=\left(p_{i}+p_{j}\right)^{2}$, then one is in the collinear regime. In this regime, the matrix element squared is evaluated in the leading pole approximation and the integration near the collinear pole is done analytically. The cross section contribution is de facto $2 \rightarrow 2$, and it is combined with the leading order and virtual contributions to the $2 \rightarrow 2$ subprocesses. If a final state gluon energy (in the subprocess rest frame) has value $E_{g}<\delta_{s} \sqrt{s_{12}} / 2$, then one is in the soft regime. The integrations of the squared matrix elements are performed analytically using the soft gluon approximation, and combined with contributions from $2 \rightarrow 2$ subprocesses. The total $2 \rightarrow 2$ results, after factorization, are finite, but depend on $\delta_{s}$ and $\delta_{c}$, such that the soft and collinear singularities are recovered in the $\delta \rightarrow 0$ limit. The remaining phase space integrations are performed via Monte Carlo. This allows easy binning of any desired observables and allows for the simple evaluation of the effect of experimental cuts on the NLO prediction [7].15]. The $2 \rightarrow 3$ contributions are all positive definite over phase space, but are also singular as $\delta_{s} \rightarrow 0$ or $\delta_{c} \rightarrow 0$. The $2 \rightarrow 2$ contributions compensate the $2 \rightarrow 3$ contributions and result in a total cross section which is independent of $\delta_{s}$ and $\delta_{c}$ over a wide range of values [7]. The expressions for all $2 \rightarrow 2$ and $2 \rightarrow 3$ processes in direct photon production, through NLO, are compiled in Ref. [7]. This is the starting point of our evaluation of the transverse momentum of the direct photon using a merger of NLO QCD and parton showers.

The PS algorithm combines the simplified collinear dynamics, represented by the $Q^{2}$ evolution of parton distribution functions and fragmentation functions, with the exact kinematics of multiple parton emission [12,13]. As implemented here, no additional weights to the integral are included with parton showers, as the $Q^{2}$ evolved distribution functions and fragmentation functions are used in evaluating the differential cross section. For the direct photon transverse momentum distribution, initial rather than final state showering is most important. Using a backward shower algorithm [13], the initial state showers are evolved backward from a starting virtuality $t_{v}$. The kinematics of the multiple partons in the initial state shower result in transverse momenta for the partons participating in the

hard scattering, effectively boosting the direct photon transverse momentum relative to the 
collinear approximation of the kinematics. In practice, the parton shower is cutoff at some low $t_{\min }$ value where perturbative QCD is still valid, but where the multiple emissions no longer become resolvable. In all the results described below, we set $t_{\min }=5 \mathrm{GeV}^{2}$. Different prescriptions have been worked out for modeling final state showers [12] as opposed to initial state (backward) showers [13]. At this stage, in a full simulation, the explicit parton emissions would be combined with a hadronization model which converts the partons into detectable particles. Our calculation does not include hadronization. The inclusion of hadronization should not alter our conclusion that multiple parton emission in the initial state can qualitatively account for the discrepancy between theory and experiment in direct photon production.

While the PS prescription for LL calculations is straightforward, the prescription for merging PS with NLO calculations is not. One problem is that the shower emission from a $2 \rightarrow 2$ subprocess may be double counted by the exact emission of an extra parton in the $2 \rightarrow 3$ subprocess. Another problem is that, to be consistent, NLO dynamics should be used to govern the parton shower development. We use initial and final state shower algorithms consistent with LL dynamics, although we use the NLO parton distribution functions in our calculation of initial state shower probabilities. Consequently, our calculation is not consistent to NLO: the PS algorithm here should be regarded only as a parametrization of a fully consistent NLO PS program. From a practical standpoint, the error induced by using only collinear dynamics in the PS algorithm in the first place should be far larger than the error induced by neglecting NLO corrections to the underlying collinear shower dynamics. Our goal here is to demonstrate that multiple parton emissions may be responsible for the discrepancy between data and theory at low transverse momentum.

To avoid the double counting problem, we restrict shower development to the $2 \rightarrow 3$ subprocesses in which all momentum vectors are large and well separated. One can view a Monte Carlo NLO calculation as a sort of truncated parton shower, with only a single extra parton emission, but which is performed exactly to $\mathcal{O}\left(\alpha \alpha_{s}^{2}\right)$. In this case, the $2 \rightarrow 2$ contributions, which include various soft and collinear terms for which the starting shower virtuality would be tiny, would never shower. If the starting shower virtuality is appropriately chosen for the $2 \rightarrow 3$ subprocesses, then only energetic, well-separated 3-body final states will develop a parton shower. Thus, the third parton of the $2 \rightarrow 3$ subprocess can be viewed as the first of the potentially multiple emissions, but which is performed using exact instead of collinear dynamics.

In our calculation of direct photon production, we have started with the NLO calculation of Ref. [7] merged with the PS along the lines of the preceding discussion. Our computer program generates $2 \rightarrow 2$ subprocesses, which frequently have negative weights, along with $2 \rightarrow n$ processes, with positive definite weights, but where $n \geq 3$. Crucial to our calculation is the stipulation of the starting virtualities for the parton shower.

A naive choice of starting virtuality $t_{v}$, such as $\left|t_{v}\right|=n p_{T}^{2}(\gamma)$, (with $n \sim 1$ ) does not ensure that the 3-parton final state is well separated. This choice leads to large amounts of showering even for soft or collinear configurations. One example of allowed showering with $\left|t_{v}\right|=n p_{T}^{2}$ is a high $p_{T}$ photon recoiling against two nearly collinear partons, with $\left|t_{45}\right|>\delta_{c} s_{12}$ but still small. This is a region of phase space where the $2 \rightarrow 2$ and $2 \rightarrow 3$ contributions at a specific $p_{T}(\gamma)$ may cancel. Since showering is implemented only in the $2 \rightarrow 3$ processes and may result in a boosted $p_{T}(\gamma)$ for the $2 \rightarrow 3$ contribution, the required cancellation may 
not occur. This introduces a dependence on $\delta_{c}$ (and $\delta_{s}$ for other configurations) which is

unphysical. In our procedure for merging NLO with PS, we minimize (but never completely eliminate) the dependence of results on variations of parameters.

To minimize the dependence of results on $\delta_{s}$ and $\delta_{c}$, we set the starting virtuality for initial state partons to $\left|t_{v}\right|=c_{v} \min \left(\left|t_{i j}\right|, s_{i j}\right)$ for $i, j=1-5$, namely, the minimum of all invariants formed by the five momenta in the $2 \rightarrow 3$ process, up to a multiplicative constant $c_{v}$. With this prescription, any nearly soft or collinear emissions in the $2 \rightarrow 3$ subprocess will result in small starting virtualities, and a small probability to shower. Only energetic, well separated $2 \rightarrow 3$ subprocesses will develop a significant parton shower in the initial state. The final state showers are initiated with starting virtuality $s_{12}$. Final state showers do not change $p_{T}(\gamma)$ relative to the unshowered calculation; they can, however, affect the number of final state photons passing the isolation cut.

\section{CALCULATIONAL RESULTS AND COMPARISON WITH DATA}

Direct photon production data from a variety of fixed target and collider experiments have been tabulated as a function of $x_{T}(\gamma)=2 p_{T}(\gamma) / \sqrt{s}$ in two recent studies 8, 10]. To compare against NLO calculations, it has proven convenient to plot the quantity (Data - Theory)/Theory. Thus, data in perfect agreement with theory would lie along the $y=0$ horizontal line. In Ref. [8], a common trend amongst the various experimental data sets was noticed, when compared against NLO QCD. For almost all data sets tabulated, the low $x_{T}(\gamma)$ range was underestimated by the theory (NLO QCD). In Ref. [10], the authors were able to improve somewhat the data vs. theory discrepancy by adjusting independently the factorization and renormalization scales. Nevertheless, the discrepancy between data and theory persists.

In Fig. 1a, we show (Data - NLO)/NLO vs. $x_{T}(\gamma)$ for data from the CDF experiment 16] at the Fermilab Tevatron, using $p \bar{p}$ collisions at $\sqrt{s}=1.8 \mathrm{TeV}$. The data points are taken from Ref. [8], where the NLO distributions are calculated using the CTEQ2M PDF's [17] evaluated at the renormalization/factorization scale $\mu=p_{T}(\gamma)$. The large enhancement of data over theory can be seen below $x_{T}(\gamma) \sim 0.05$, which corresponds to $p_{T}(\gamma) \lesssim 45 \mathrm{GeV}$ at the Tevatron. Our calculation employs the same scale choices as Ref. [8], but updated CTEQ3M PDF's 18]. In keeping with CDF cuts, we require the photon pseudorapidity $|\eta(\gamma)|<0.9$, and a photon isolation cut which requires that the sum of energy, projected transverse to the beam axis, $\left(E_{T}^{i}\right)$ of parton $i$ within a cone of size $\Delta R=\sqrt{(\Delta \eta)^{2}+(\Delta \phi)^{2}}=$ 0.7 satisfy

$$
\left.\sum_{i} E_{T}^{i}\right|_{\Delta R=0.7}<2 \mathrm{GeV} .
$$

These two cuts are also used in Figs. 2 and 3 below.

To minimize differences due to parton distribution choices, etc., rather than comparing the data to our NLO calculation merged with parton showers (NLO $\oplus \mathrm{PS}$ ), we show the effect of showering as an excess or deficit relative to the unshowered NLO calculation. In Figs. $1 b$ and $1 c$, we show the relative $x_{T}(\gamma)$ distributions (NLO $\oplus$ PS-NLO)/NLO where the initial state virtuality is chosen with $c_{v}=4$. In our calculation, we have run for subprocess photon $p_{T}(\gamma)>4 \mathrm{GeV}$, since the matrix elements are singular as $p_{T}(\gamma) \rightarrow 0$; the results do not 
change noticeably if instead we use $p_{T}(\gamma)>2 \mathrm{GeV}$. Fig. $1 b$ employs $\delta_{s}=10 \delta_{c}=0.1$, and Fig. $1 c$ has $\delta_{s}=10 \delta_{c}=0.02$. We see in Figs. $1 b$ and $1 c$ that the incorporation of the PS has led to an enhancement of the relative $x_{T}(\gamma)$ distributions at $x_{T}(\gamma) \sim 0.02$ of about $30-40 \%$, and hence is in accord with the data for the low range of $x_{T}(\gamma)$. The enhancement has been traced to the fact that a small fraction of the large population of very low $x_{T}(\gamma)$ photons gets boosted up to higher energies by recoiling against the multiple parton emissions. Although the enhancement at low $x_{T}(\gamma)$ from the NLO $\oplus \mathrm{PS}$ calculation is similar for the two cases, the large relative $x_{T}(\gamma)$ distributions show a deficit of $10-20 \%$. The high $x_{T}(\gamma)$ deficit is due to the effect of the photon isolation cut.

For very high energy events, there can still exist significant shower virtualities for events with quasi-soft or collinear partons, which introduces a slight dependence on $\delta_{s}$ and $\delta_{c}$. There is some enhancement in showering for very high energy events, which leads to fewer isolated photons, and a net diminution of signal due to the isolation cut.

If we modify the initial shower virtuality magnitude by varying $c_{v}$, we find that a choice of $c_{v} \sim 1$ results in modest enhancements of the low $x_{T}(\gamma)$ region by only $\sim 10 \%$. Choosing $c_{v}$ as high as $c_{v} \simeq 9$ yields enhancements typically around $80 \%$. Also, we have investigated how the results change by changing the initial state shower cutoff virtuality choice from $t_{\text {min }}=5 \mathrm{GeV}^{2}$ to $t_{\min }=3 \mathrm{GeV}^{2}$. The latter variation yields typically a $20 \%$ effect. In spite of these various uncertainties, the overall qualitative trend of enhanced cross section at $x_{T}(\gamma) \lesssim 0.06$ persists in all the cases we have examined.

In Ref. [8], it was noted that an ad-hoc Gaussian smearing of the subprocess $p_{T}$ led to improved agreement between theory and data. In Fig. 1d, we additionally introduce Gaussian smearing (GS) to both $2 \rightarrow 2$ and $2 \rightarrow 3$ processes, with average transverse momentum zero and width $\sigma=1 \mathrm{GeV}$. The overall enhancement of the NLO $\oplus \mathrm{PS}$ at $x_{T}(\gamma) \sim$ 0.02 remains, but with some slight additional enhancement for $\mathrm{NLO} \oplus \mathrm{PS} \oplus \mathrm{GS}$ at even lower $x_{T}(\gamma)$ values. The small effect of the Gaussian smearing at CDF is not surprising since the average boost generated by the PS algorithm is $\sim 2.5 \mathrm{GeV}$.

In Fig. $2 a$, we show data from the UA2 experiment [19] ( $p \bar{p}$ collisions at $\sqrt{s}=630$ $\mathrm{GeV}$ ) compared with NLO QCD, for scale choice $\mu=p_{T}(\gamma) / 2$. Here we use a photon $p_{T}$ cutoff of $p_{T}(\gamma)>2 \mathrm{GeV}$. Again, we see that data exceeds theory by $\sim 40 \%$, although this time for $x_{T}(\gamma) \sim 0.05$ (corresponding to $p_{T}(\gamma) \sim 16 \mathrm{GeV}$ ). In Fig. $2 b$, we plot the $\mathrm{NLO} \oplus \mathrm{PS}$ result, using again the initial state virtuality choice $c_{v}=4$, and for $\mu=p_{T}(\gamma) / 2$ and $\delta_{s}=10 \delta_{c}=0.02$. Our merged NLO $\oplus \mathrm{PS}$ calculation gives an enhancement of $\sim 20 \%$ above NLO results for $x_{T}(\gamma) \sim 0.05$. Although the CDF and UA2 calculations start with similar virtualities, the relatively higher value of Feynman- $x$ in the UA2 case leads to lesser amounts of initial state PS radiation. This can be offset to some extent by choosing a higher starting virtuality, $c_{v}=9$, shown in Fig. $2 c$. The increase in virtuality leads to a rise in our calculation to about $40 \%$ above NLO expectations, in accord with the data. Finally, in Fig. $2 d$, we include as well the Gaussian smearing, which leads to some additional enhancement at low $x_{T}(\gamma)$.

Finally, we turn to much lower energy $p p$ collider results from experiments at the CERN ISR at $\sqrt{s}=63 \mathrm{GeV}$. In Fig. 3a, we show the data from the R806 experiment [20] compared with NLO QCD for $\mu=p_{T}(\gamma) / 2$. Using the same scale $\mu$, and including parton showers, we show in Figs. $3 b$ and $3 c$, the comparison (NLO $\oplus \mathrm{PS}-\mathrm{NLO}) / \mathrm{NLO}$ for $c_{v}=4$ and $c_{v}=9$, respectively. We have lowered the photon $p_{T}$ cutoff here to $p_{T}(\gamma)>1 \mathrm{GeV}$. Because of the 
large values of parton $x$ and small virtualities, at this energy, there is very little showering, so that perturbative multiple parton emission as described by the PS algorithm cannot explain the data/theory discrepancy. However, Gaussian smearing on the order of $1 \mathrm{GeV}$ can be a large effect at this energy, where $x_{T}(\gamma)=0.1-0.4$ corresponds to $p_{T}(\gamma)=3-13 \mathrm{GeV}$. In Fig. 3d, we invoke as usual the $\sigma \sim 1 \mathrm{GeV}$ Gaussian smearing of the subprocess transverse momentum. In this case, the smearing can move the low $x_{T}(\gamma)$ theoretical prediction into rough agreement with the data.

\section{SUMMARY AND CONCLUSIONS}

In summary, we have investigated the effects of multiple parton emissions on direct photon production in hadronic collisions by merging the PS technique with NLO QCD. For experiments at very high energy (e.g. UA2 and CDF), the extra $k_{T}$ smearing of the hard scattering subprocess induced by the multiple parton emissions can cause some of the relatively numerous low $p_{T}$ photons from NLO QCD to be boosted to higher $p_{T}$ values. Such an effect causes a shift in the predicted $x_{T}(\gamma)$ distribution, thereby improving the agreement between theory and experiment. Our results cannot be interpreted as a QCD prediction due to the many uncertainties in the PS algorithm, and in our merging procedure. Amongst these uncertainties are the nature of the PS algorithm itself, and the prescription for initial and cutoff virtualities in the PS. On the other hand, our results can be interpreted as an existence proof that higher order effects (particularly from multiple parton emission) can account for the theory vs. data discrepancy. Other groups 10,11 have noted that the theory vs. data discrepancy can be resolved in NLO QCD mainly by using modified parton distribution functions. We comment that our result of an appropriately shifted $x_{T}(\gamma)$ distribution will obtain for any choice of PDF's or hard scattering scale choices, as long as sufficient parton showering can be produced. Since hard scattering processes in nature are of course all-orders processes, one would expect at some level a discrepancy between data and fixed order QCD to occur. Our results show that this may already be the case for the direct photon $x_{T}(\gamma)$ distributions.

For lower energy data sets $(e . g \cdot \sqrt{s} \lesssim 63 \mathrm{GeV})$, it is difficult to produce sufficient QCD radiation via the PS to improve the theory vs. data discrepancy. We do note, as in Ref. [8], that an intrinsic Gaussian $k_{T}$ smearing with width $\sigma \sim 1 \mathrm{GeV}$ will push the theory in the right direction to match with data. Thus, the theory vs. data discrepancy can be resolved globally by invoking extra $k_{T}$ for the hard scattering partons: that $k_{T}$ would be primarily perturbative in nature for high energy data sets, but mainly non-perturbative for data sets taken at $\sqrt{s} \lesssim 100 \mathrm{GeV}$.

\section{ACKNOWLEDGMENTS}

We thank J. Huston, S. Kuhlmann and J. F. Owens for discussions. This research was supported in part by the U. S. Department of Energy under grant number DE-FG-0587ER40319 and the National Science Foundation Grants PHY 93-07213 and PHY 95-07688. 


\section{REFERENCES}

[1] F. Halzen, M. Dechantsreiter and D. M. Scott, Phys. Rev. D22, 1617 (1980); F. Halzen and D. M. Scott, Phys. Rev. D18, 3378 (1978); F. Halzen and D. M. Scott, Phys. Rev. Lett. 40, 1117 (1978).

[2] A. P. Contogouris, N. Mebarki, E. N. Argyres and S. D. P. Vlassopulos, Phys. Rev. D35, 1584 (1987); E. N. Argyres, A. P. Contogouris, M. Sanielevici and H. Tanaka, Phys. Rev. D29, 2527 (1984); A. P. Contogouris, S. Papadopoulos and J. Ralston, Phys. Lett. 104B, 70 (1981).

[3] L. Cormell and J. F. Owens, Phys. Rev. D22, 1609 (1980).

[4] E. Berger, E. Braaten and R. Field, Nucl. Phys. B239, 52 (1984).

[5] For a review, see J. F. Owens, Rev. Mod. Phys. 59, 465 (1987).

[6] P. Aurenche, R. Baier, A. Douiri, M. Fontannaz and D. Schiff, Nucl. Phys. B286, 553 (1987).

[7] H. Baer, J. Ohnemus, and J. F. Owens, Phys. Lett. 234B, 127 (1990) and Phys. Rev. D42, 61 (1990).

[8] J. Huston, E. Kovac, S. Kuhlmann, H. Lai, J. F. Owens and W. K. Tung, Phys. Rev. D51 6139 (1995).

[9] M. Gluck, L. E. Gordon, E. Reya, W. Vogelsang, Phys. Rev. Lett. 73, 388 (1994).

[10] W. Vogelsang and A. Vogt, Nucl. Phys. B453 334 (1995).

[11] E. Quack and D. K. Srivastava, GSI-94-40 (1995).

[12] G. Fox and S. Wolfram, Nucl. Phys. B168, 285 (1980) and R. Odorico, Nucl. Phys. B172, 157 (1980).

[13] T. Sjöstrand, Phys. Lett. 157B, 321 (1985); T. Gottschalk, Nucl. Phys. B277,700 (1986).

[14] H. Baer and M. H. Reno, Phys. Rev. D44, R3375 (1991) and Phys. Rev. D45, 1503 (1992)

[15] H. Baer, J. Ohnemus and J. F. Owens, Phys. Rev. D40, 2844 (1989).

[16] F. Abe et. al., (CDF Collaboration), Phys. Rev. Lett. 68, 2734 (1992) and Phys. Rev. D48, 2998 (1993).

[17] J. Botts et. al., Phys. Lett. B304, 159 (1993).

[18] H. L. Lai et. al., Phys. Rev. D51, 4763 (1995).

[19] J. Alitti et. al., (UA2 Collaboration), Phys. Lett. B263, 544 (1991).

[20] E. Anassontzis et. al., (R806 Collaboration), Z. Phys. C13, 277 (1982). 


\section{FIGURES}

FIG. 1. $x_{T}(\gamma)$ distribution for $p \bar{p}$ collisions at $\sqrt{s}=1.8 \mathrm{TeV}$. We show $a$ ) (Data-NLO)/NLO for $\mathrm{CDF}$ data, $b)(\mathrm{NLO} \oplus \mathrm{PS}-\mathrm{NLO}) / \mathrm{NLO}$ for $\left.\delta_{s}=10 \delta_{c}=0.1, c\right)(\mathrm{NLO} \oplus \mathrm{PS}-\mathrm{NLO}) / \mathrm{NLO}$ for $\delta_{s}=10 \delta_{c}=0.02$, and $\left.d\right)(\mathrm{NLO} \oplus \mathrm{PS} \oplus \mathrm{GS}-\mathrm{NLO}) / \mathrm{NLO}$ for $\delta_{s}=10 \delta_{c}=0.02$. For all plots, the hard scattering $p_{T}(\gamma) \geq 4 \mathrm{GeV}$.

FIG. 2. $x_{T}(\gamma)$ distribution for $p \bar{p}$ collisions at $\sqrt{s}=0.63 \mathrm{TeV}$. We show a) (Data-NLO)/NLO for $\mathrm{UA} 2$ data, $b)(\mathrm{NLO} \oplus \mathrm{PS}-\mathrm{NLO}) / \mathrm{NLO}$ for $\left.c_{v}=4, c\right)(\mathrm{NLO} \oplus \mathrm{PS}-\mathrm{NLO}) / \mathrm{NLO}$ for $c_{v}=9$, and d) $(\mathrm{NLO} \oplus \mathrm{PS} \oplus \mathrm{GS}-\mathrm{NLO}) / \mathrm{NLO}$ for $c_{v}=9$. For all plots, the hard scattering $p_{T}(\gamma) \geq 2 \mathrm{GeV}$ and $\delta_{s}=10 \delta_{c}=0.02$.

FIG. 3. $x_{T}(\gamma)$ distribution for $p p$ collisions at $\sqrt{s}=63 \mathrm{GeV}$. We show $a$ ) (Data-NLO)/NLO for $\mathrm{R} 806$ data at the CERN ISR, $b)(\mathrm{NLO} \oplus \mathrm{PS}-\mathrm{NLO}) / \mathrm{NLO}$ for $\left.c_{v}=4, c\right)(\mathrm{NLO} \oplus \mathrm{PS}-\mathrm{NLO}) / \mathrm{NLO}$ for $c_{v}=9$, and $\left.d\right)(\mathrm{NLO} \oplus \mathrm{PS} \oplus \mathrm{GS}-\mathrm{NLO}) / \mathrm{NLO}$ for $c_{v}=9$. For all plots, the hard scattering $p_{T}(\gamma) \geq 1$ $\mathrm{GeV}$ and $\delta_{s}=10 \delta_{c}=0.1$. 

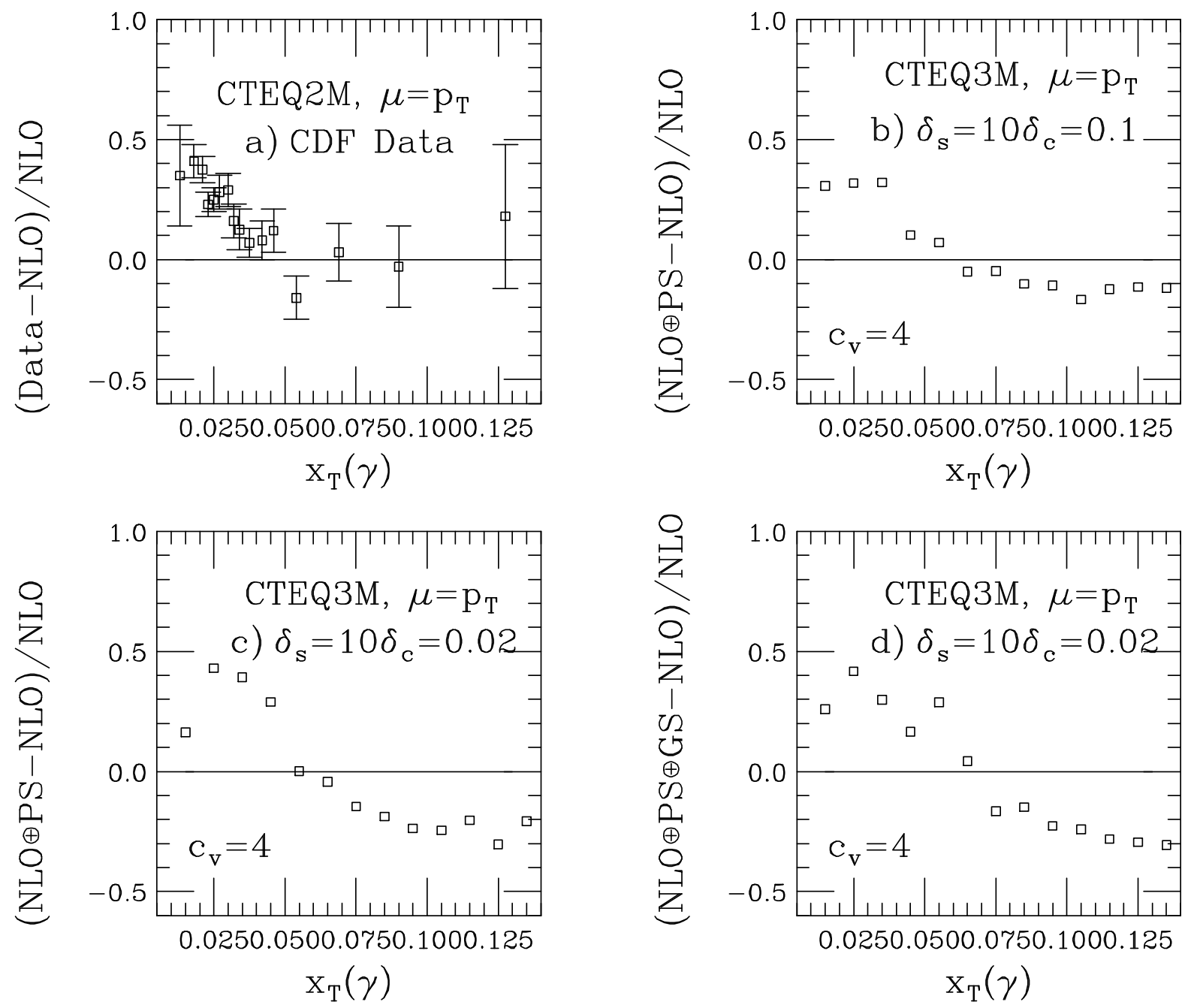

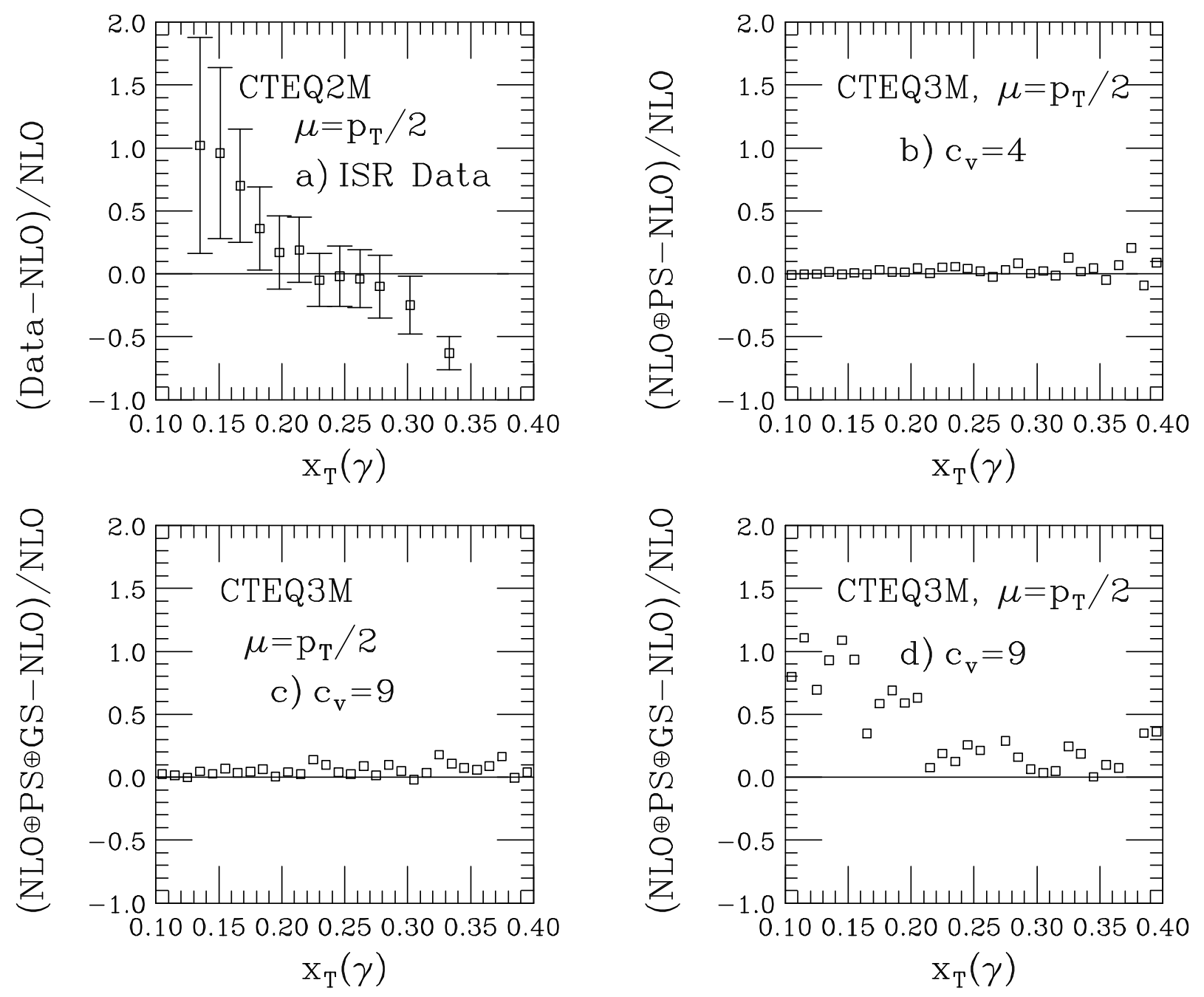

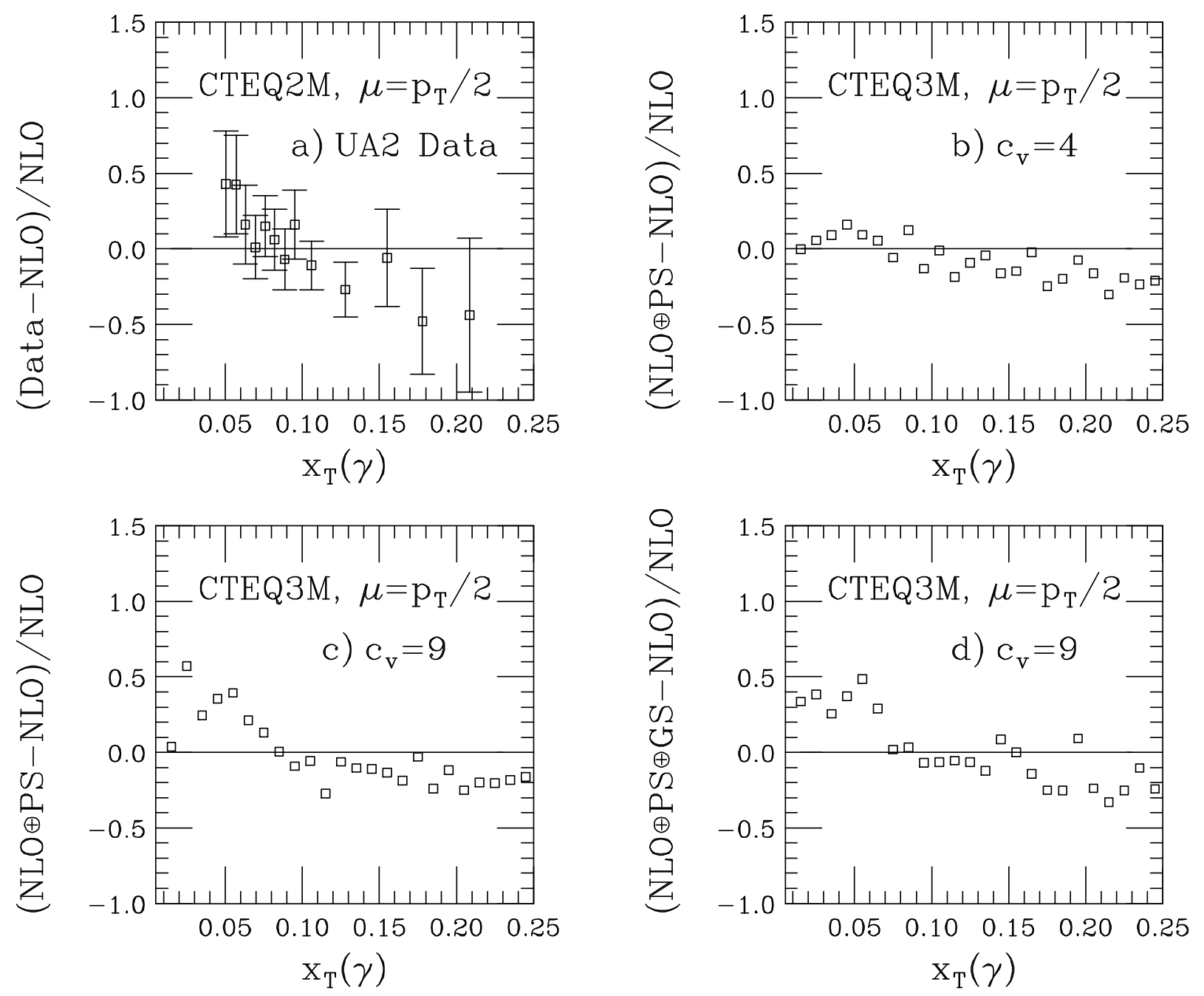